\title{
Dynamic Simulation of Pump-Storage Power Plants with different variable speed configurations using the Simsen Tool
}

\author{
Klaus Krüger and Jiri Koutnik \\ Voith Hydro Holding GmbH \& Co. KG, \\ P.O. Box 2010, 89510 Heidenheim, Germany \\ Klaus.Krueger@voith.com, Jiri.Koutnik@voith.com
}

\begin{abstract}
Pumped storage power plants are playing a significant role in the contribution to the stabilization of an electrical grid, above all by stable operation and fast reaction to sudden load respectively frequency changes. Optimized efficiency and smooth running characteristics both in pump and turbine operation, improved stability for synchronization in turbine mode, load control in pump mode operation and also short reaction times may be achieved using adjustable speed power units. Such variable speed power plants are applicable for high variations of head (e.g. important for low head pump-turbine projects). Due to the rapid development of power semiconductors and frequency converter technology, feasible solutions can be provided even for large hydro power units. Suitable control strategies as well as clear design criteria contribute significantly to the optimal usage of the pump turbine and motor-generators.
\end{abstract}

The SIMSEN tool for dynamic simulations has been used for comparative investigations of different configurations regarding the power converter topology, types of semiconductors and types of motor-generators including the coupling to the hydraulic system. A brief overview of the advantages \& disadvantages of the different solutions can also be found in this paper. Using this approach, a customized solution minimizing cost and exploiting the maximum usage of the pump-turbine unit can be developed in the planning stage of new and modernization pump storage projects.

Keywords: pumped storage, pump-turbine, variable speed, dynamic simulation, optimization, power converters.

\section{Introduction}

For the final decision regarding the appropriate combination of power semiconductors and frequency converter variant (short: power converter) and motor-generator type following aspects have to be considered simultaneously, as they influence each other:

- $\quad$ overall arrangement (e.g. space requirements in a cavern),

- $\quad$ speed variability range,

- variations in head,

- $\quad$ keeping maximum turbine efficiency over the whole operating range,

- low pressure pulsations and power oscillations,

- $\quad$ fast reaction times for active power control (load ramping and spinning reserve),

- $\quad$ possibility and range of reactive power control,

- $\quad$ amount of Total Harmonic Distortion (THD) fed into the grid, which may require the necessity of AC filters (THD refers in this case to higher harmonic components in voltage \& current),

- $\quad$ losses in power converter versus motor-generator losses, 
intended load control band in pump-mode operation (e.g. important in case of volatile power generation like upcoming large wind farms),

- $\quad$ additional safety margin from the pump instability region.

While from the above list turbine design [1] and hydraulic layout aspects are important considerations, this paper will give an introduction about power converter applications for achieving the variable speed ability for power units. First of all this paper gives an overview of different possibilities for pump storage plants with variable speed, which should be considered in the planning stage of a project. Furthermore the benefits of a combined dynamic simulation of the hydraulic system coupled with the electric system including the turbine controller and the controller influencing the electrical machine and power converters are figured out as a prerequisite for the above mentioned considerations.

\section{Methods of Simulations}

In order to explore an optimum solution, the behaviour of the pump-turbines with variable speed may be simulated principally on 2 levels:

a) simulation of the electrical part only including the motor-generator, power converter and the detailed control structure for the converter controller, however neglecting turbine characteristics and the hydraulic system dynamic properties, or

b) simulation of the complete power train starting from waterways up to the electrical high voltage grid.

The simulation tool SIMSEN [2,3] and its hydro-specific module library used at Voith Hydro allows both types of simulation, taking into account an ideal power converter behaviour, which is however sufficient for prediction of frequencies and amplitudes of higher harmonics caused by the power converters itself [4] or by non-linear consumers connected to the grid. This is decisive for estimation of additional losses in the electrical machine and influences the design of active or passive filters (if necessary).

Using this tool many aspects mentioned in the introduction can be studied for configurations with standard synchronous motor-generators using current converters connected to the stator terminals as well as DFIMs (double-fed induction machine) with power converters connected to their rotor windings.

In order to be able to evaluate above all the dynamic behaviour of the complete power unit, an extensive simulation comprising the motor-generator (in some cases including hydraulic system with turbine as well), turbine controller and the DC / AC excitation including controls is compulsory. If the voltage / current waveform distortion is of importance or if the total harmonic distortion (THD) are of interest, also the power converters including their control strategy have to be considered in detail as well. The maximum values of THD in voltage \& current signals are limited by national \& international electrical grid standards. Such higher harmonic content on the electrical currents are also a source of additional losses in the motor-generator, and have to be considered in the design of the electrical machine, even if the THD fed into the grid are below the values mentioned in the standards.

\section{Main Configurations of Variable Speed Units}

There are two basic configurations for variable speed units using the technology currently available on the market. The first group consists of a standard synchronous motor-generator with a DC static rotor excitation. The stator terminals are connected to the step-up transformer either via a so called Load Commutated Inverter or via a Self Commutated Inverter (LCI or SCI), which have to be designed for the nominal apparent power of the unit, e.g. PSP Forbach [5] (see simulation variants V1 (fig: 1) and V2 (fig. 2) in the list below).

The second option is to use a configuration consisting of a double-fed asynchronous (induction) machine with a 3-phase rotor winding. These 3 rotor windings are fed from a variable frequency voltage source, which can be a cyclo-converter (e.g. PSP Goldisthal) or a Voltage Source Inverter (VSI), for example PSP Okukiyotsu. Both variants of variable frequency voltage sources are designed for lower power than the nominal apparent power of the unit (approx. 30\%). All mentioned types of power converters exist in several variants depending on specific technical requirements and are based on different power electronic technologies. All technologies discussed use either Silicon Controlled Rectifiers (SCR), Gate Turn-Off Thyristors (GTO) or Integrated Gate Controlled Thyristors (IGCT).

In this chapter for the following variants the main advantages \& disadvantages will be listed for a fast and comprehensive overview:

V1: $\quad$ synchronous motor-generator with LCI6 (Load Commutated Inverter with SCRs)

V2: $\quad$ synchronous motor-generator with SCI6 (Self Commutated Inverter with IGCTs)

V3: asynchronous motor-generator (DFIM) with cyclo-converter (using SCRs)

V4: asynchronous motor-generator (DFIM) with 2 level VSI (Voltage Source Inverter using IGCT technology)

V5: asynchronous motor-generator (DFIM) with 3 level VSI NPC (Voltage Source Inverter with Neutral Point Clamped using IGCT technology) 
All these basic configurations were presented and discussed in more detail in [4] including some first simulative analysis. As an example variant V4 will be discussed more extensively in this paper based on simulations for a coupled hydraulic, mechanical and electrical system.

\section{V1 Synchronous Motor-Generator with LCI}

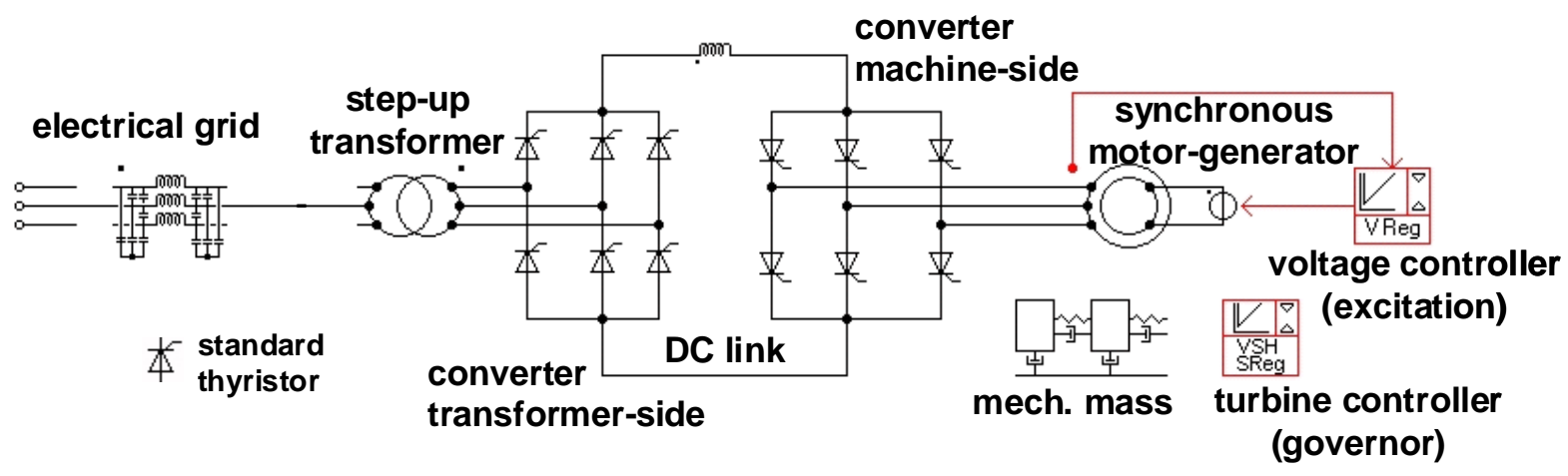

Fig. 1: Synchronous generator with Load Commutated Inverter 6 (LCI6)

In case of LCI, standard thyristors without turn-off possibility have been used (i.e. SCR technology, [5]).

\section{Advantages (+) \& Disadvantages (-) V1}

+ simple converter technology with low component count

+ can be built with SCR, simple control regime, direct influence on firing angle of thyristors

+ can be used to start-up a synchronous generator

- power converter has to be designed for total nominal motor-generator apparent power

- high total harmonic distortion (THD) at the stator / grid side $\rightarrow$ additional filters necessary

- no possibility to control reactive power flow with the electrical grid,

- motor-generator has to supply reactive power consumption of the machine-side converter $\rightarrow$ increases power rating

Expanding of LCI 6 to LCI 12 (i.e. 12 SCRs on each side are then necessary) reduces the number of harmonics, however additional costs and additional losses are caused by the converter itself and a 3x3 phase transformer and/or an additional stator winding for the electrical machine [6] are required.

\section{V2 Synchronous Motor-Generator with SCI6}

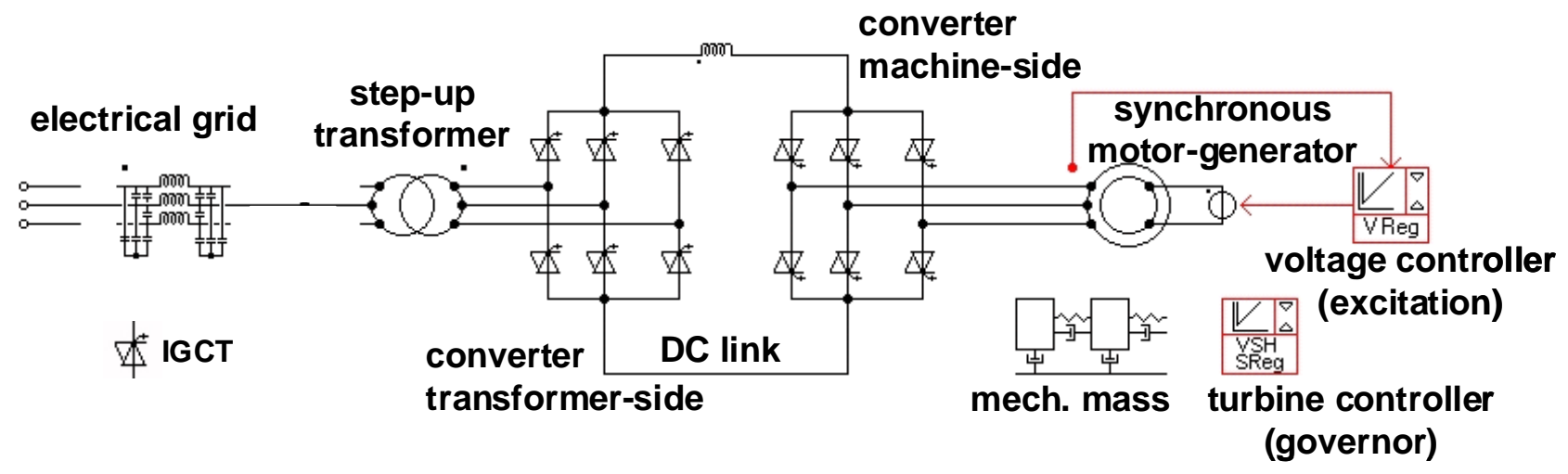

Fig. 2: Synchronous motor-generator with SCI6 configuration [7]

SCI6 means Self Commutated Inverter with 6 IGCTs on the transformer-side and machine-side converter. Different from the LCI variant, where standard thyristors without turn-off possibility have been used, SCI inverters consist of IGCT power semiconductors. This type of semiconductors allows turn-off and Pulse Width Modulation (PWM) with carrier frequency up to 1.5 $\mathrm{kHz}$. Therefore such power semiconductors in combination with an appropriate control strategy allow to build up the sinus form 
of the voltage / current on the transformer side much better than in case of LCI. The control strategy of SCI is decisive for the signal total harmonic distortion. Nowadays SCI technologies are available for technical applications from 100 MVA to approx. 200 MVA.

\section{Advantages (+) \& Disadvantages (-) V2}

+ lower voltage total harmonic distortion

+ control of reactive power flow from converter to the grid

+ lower switching losses compared to LCIs

+ available for all medium voltage ranges, minimum number of elements for given power rating

- power converter has to be designed for total nominal motor-generator apparent power

- complex control structure for firing circuits for IGCTs compared to LCIs

- higher costs of IGCT compared to SCR (approx. by factor 3)

\section{V3 Asynchronous Motor-Generator (DFIM) With Cyclo-Converter Cascade Using SCR Technology}

This configuration is not the most up-to-date solution (shown in fig. 3) for variable speed technology, as the cyclo-converter produces harmonic distortions larger than VSI variants (V4 \& V5), mostly on the rotor circuit. This can be improved by installing passive filters. However, this arrangement is presented to show its advantages / disadvantages, as some plants with this configuration are in the construction stage.

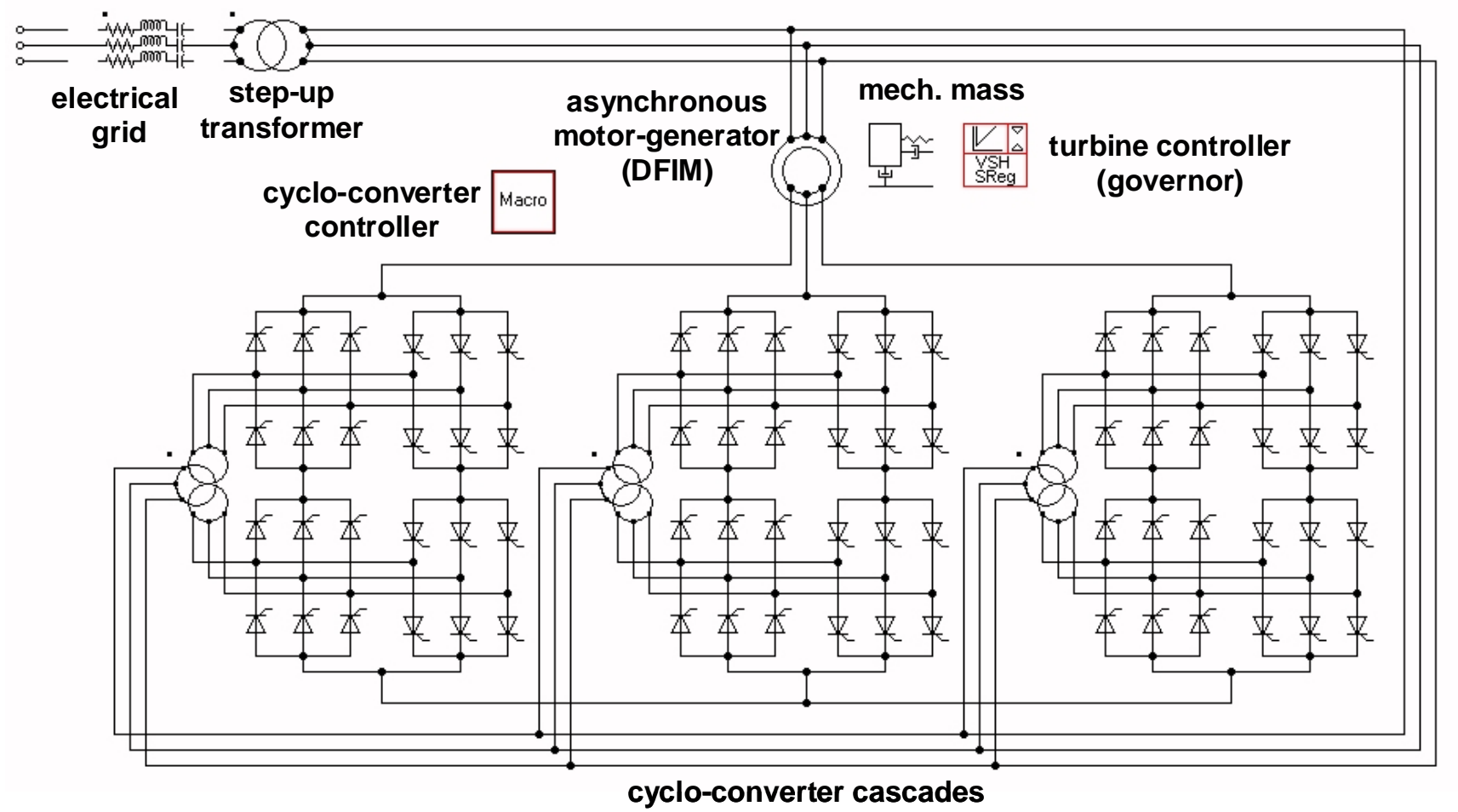

Fig. 3: DFIM with cyclo-converter cascade

\section{Advantages (+) \& Disadvantages (-) V3}

+ control of active and reactive power flow possible

+ lower total harmonic distortion at generator/grid side

+ converters to be designed only for fractional part of total nominal generator apparent power

- 3 multi-windings auxiliary transformers necessary

- complex control regime, determination and triggering of firing angle of 72 thyristors 
- high parts count $\rightarrow$ increased risk of component failure

- filters in rotor circuitry may be necessary

- additional static SFC necessary for starting in pump mode

- dominant 11th and 13th harmonics into the grid (THD)

\section{V4 Asynchronous Motor-Generator (DFIM) with 2-Level VSI using IGCT technology}

The 2-level Voltage Source Inverter (fig. 4) is an option for AC excitation of an asynchronous motor-generator with many advantages compared to the cyclo-converter variant. This has been made possible by commercial usage of IGCTs applicable for higher power, which have small switching losses and allow Pulse Width Modulation (PWM) with high switching (carrier) frequencies of more than $1 \mathrm{kHz}$. This leads to a reduction of higher harmonics into the rotor circuit (i.e. lower losses in the electrical machine) as well as into the grid [8,9]. The disadvantage is the dead band around the synchronous speed, as this type of converter is not able to supply DC voltage to the rotor circuit. This disadvantage can be avoided by using a 3-level VSI extension to an Active Neutral Point Clamped (ANPC) converter (see next Variant V5).

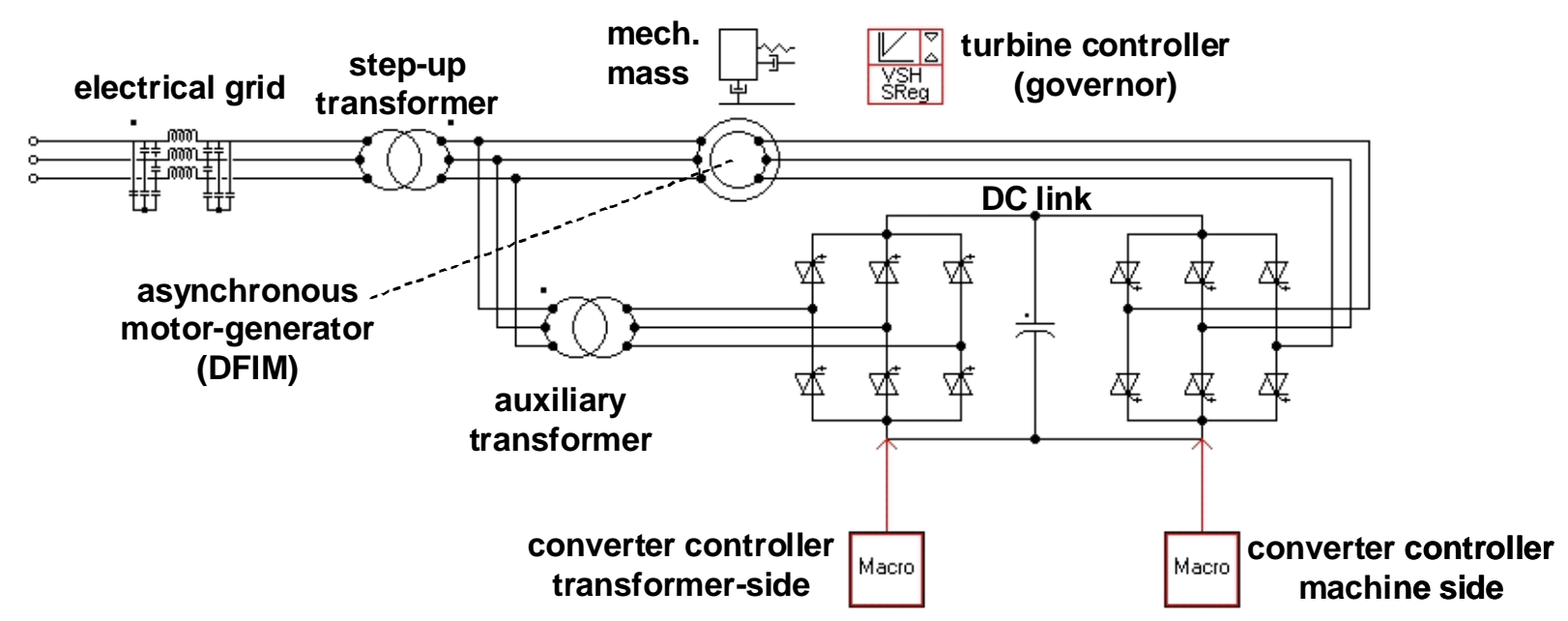

Fig. 4: Scheme of DFIM with 2-level VSI configuration

\section{Advantages (+) \& Disadvantages (-) V4}

+ converters to be designed only for fractional part of total nominal generator apparent power

+ control of active and reactive power flow possible

+ de-coupled control regimes for transformer-side and machine-side converter

+ low parts count and modular structure using simple PWM schemes

+ no SFC for start-up in pump mode necessary

+ only 1 standard auxiliary transformer needed

- multi-variable controller for transformer-side and machine-side converter necessary due to cross-influence of d- and q-axis

- higher currents total harmonic distortion (THD) compared with a 3-level VSI. Dominant harmonics into the grid depend on the PWM carrier frequency

- filters in rotor circuitry might be necessary

- can not operate at synchronous speed (dead band of $+/-2 \%$ around synchronous speed)

\section{V5 Asynchronous Motor-Generator (DFIM) with 3-Level VSI using IGCT technology}

This configuration with 3-level VSI with Neutral Point Clamp (NPC) has additional advantages compared to 2-level VSI in terms of a smoother rotor voltage waveform [10]. The principle can be seen in fig. 5 and 6.

Fig. 6 shows that the 3-level VSI solution can achieve better results regarding the "amplitude” and rotor voltage shape because it can use 3 values for the rotor voltage quantization $(0, \mathrm{U} 1 / 2, \mathrm{U} 1)$ instead of only two values (particular zero voltage and full voltage U1) in the case of a 2-level VSI. 


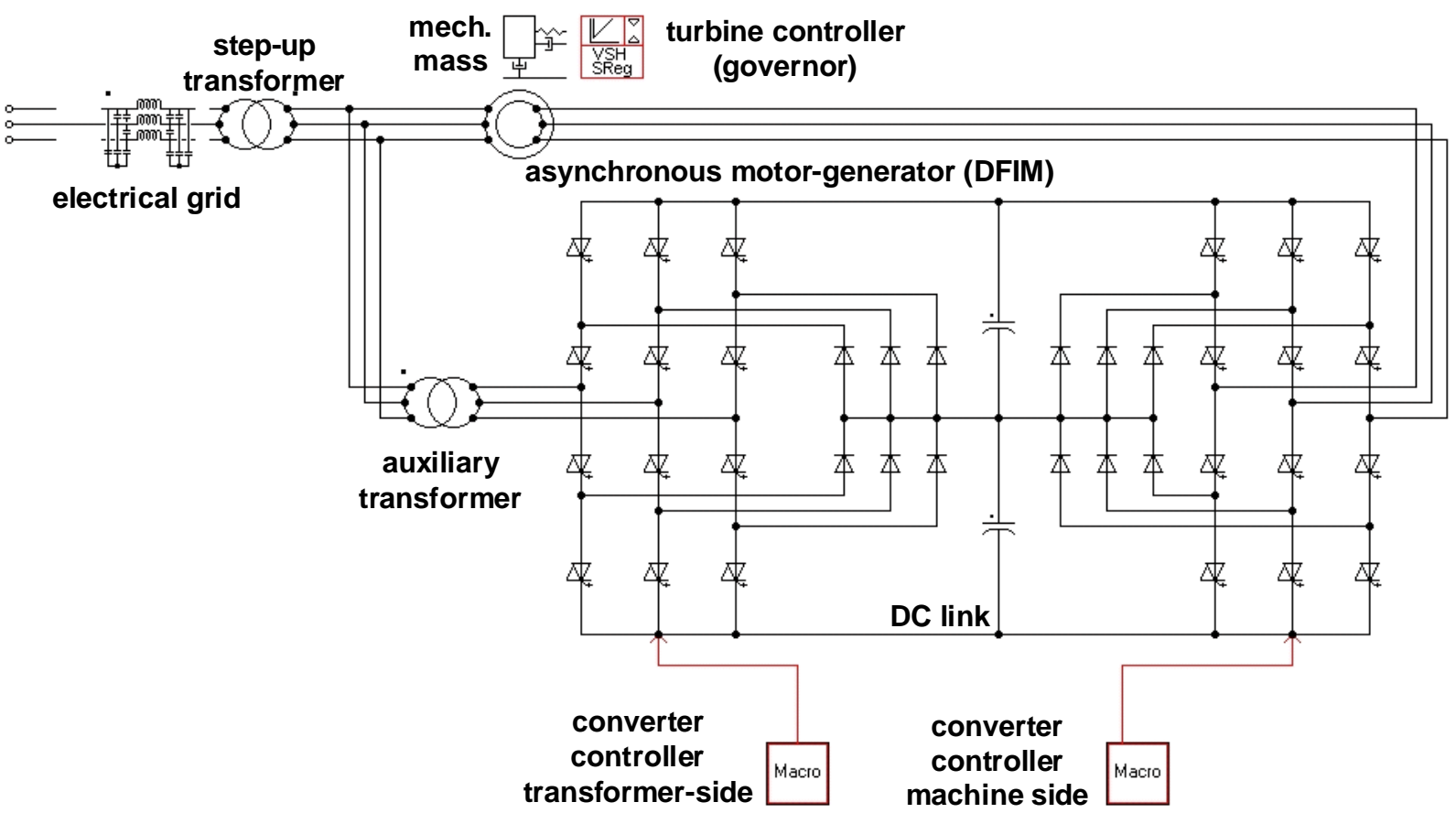

Fig. 5: Scheme of DFIM with 3-level VSI configuration

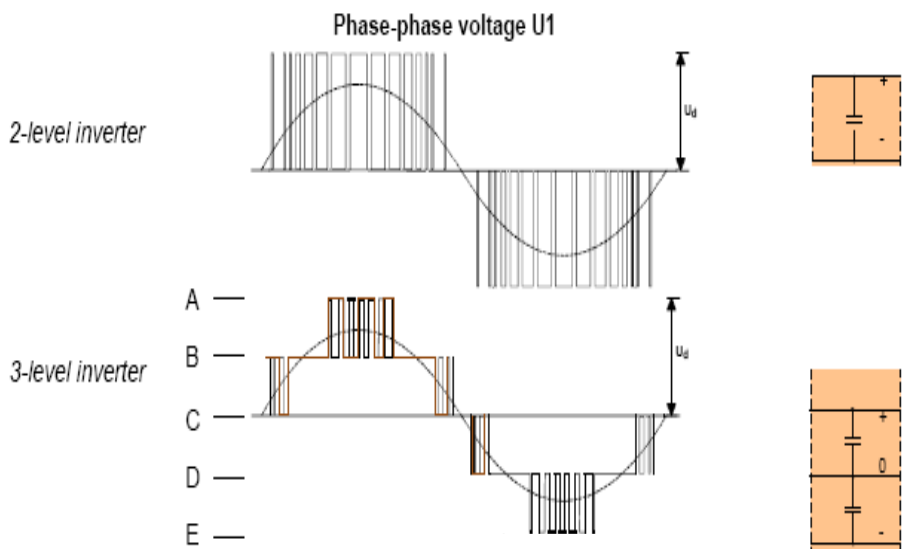

Fig. 6: Comparison of a 2- and 3-level VSI [11]

\section{Advantages (+) \& Disadvantages (-) V5}

+ see V4

+ lower current THD than the 2-level VSI [4]

+ smaller filters in rotor circuitry necessary compared with 2-level VSI

- see V4

- 4 IGCT current converters and 6 recovery diodes needed (more expensive)

The last disadvantage can be overcome for short periods of time (synchronous operation) by the extension to an Active Neutral Point Clamped (ANPC) technology using GTO instead of free-wheeling diodes indicated in fig. 5.

\section{Coupled Hydraulic \& Electrical Simulation}

For the coupled simulation the complete system "water-to-wire" has been modeled, which is shown in fig. 7. The control strategy for the transformer-side and machine-side converter can be considered as independent from each other and both are based on the Park's transformation during load operation. 


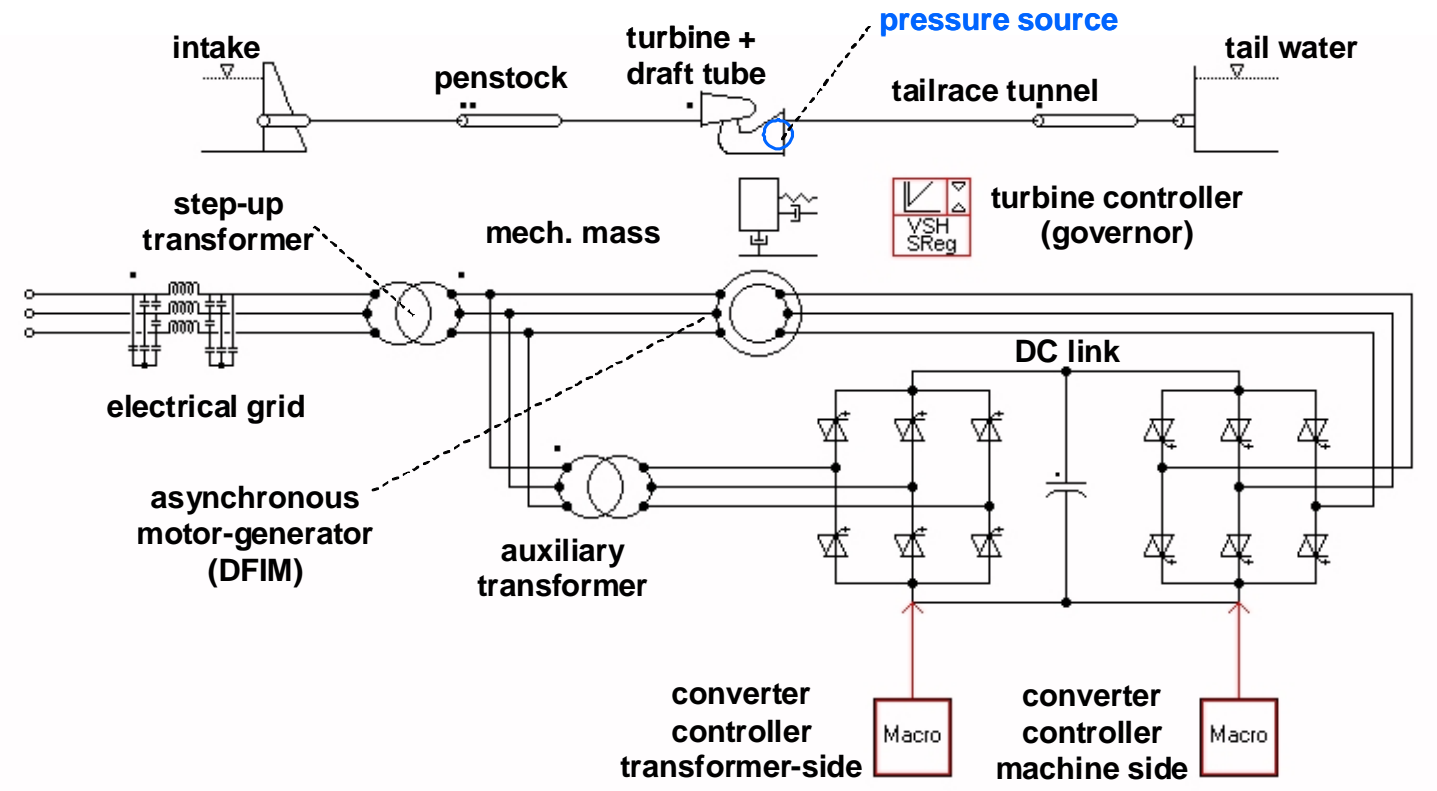

Fig. 7: Scheme of a coupled hydraulic \& electrical system for DFIM with 2-level VSI

Fig. 8 describes the over-all control structure using a double cascade control loop for the transformer-side of the VSI. The outer loop consists of two PI-controllers for the DC-link voltage and reactive power between auxiliary transformer and transformer-side converter. The setpoints of this controller are usually constant values: nominal value for the DC-voltage and zero for the reactive power between converter and auxiliary transformer. The outputs of this master controller are setpoints for the dand q-axis currents of the auxiliary transformer and the outputs of this inner controller loop are transformed back into a vector with firing impulses in order to impress the voltage in the DC-link. This control structure for the transformer-side in fig. 8 is valid for all operation conditions: start-up, synchronization, load operation and shut-down. Arrows in fig. 8 and also in fig. 9 indicate vectors, not scalar values.

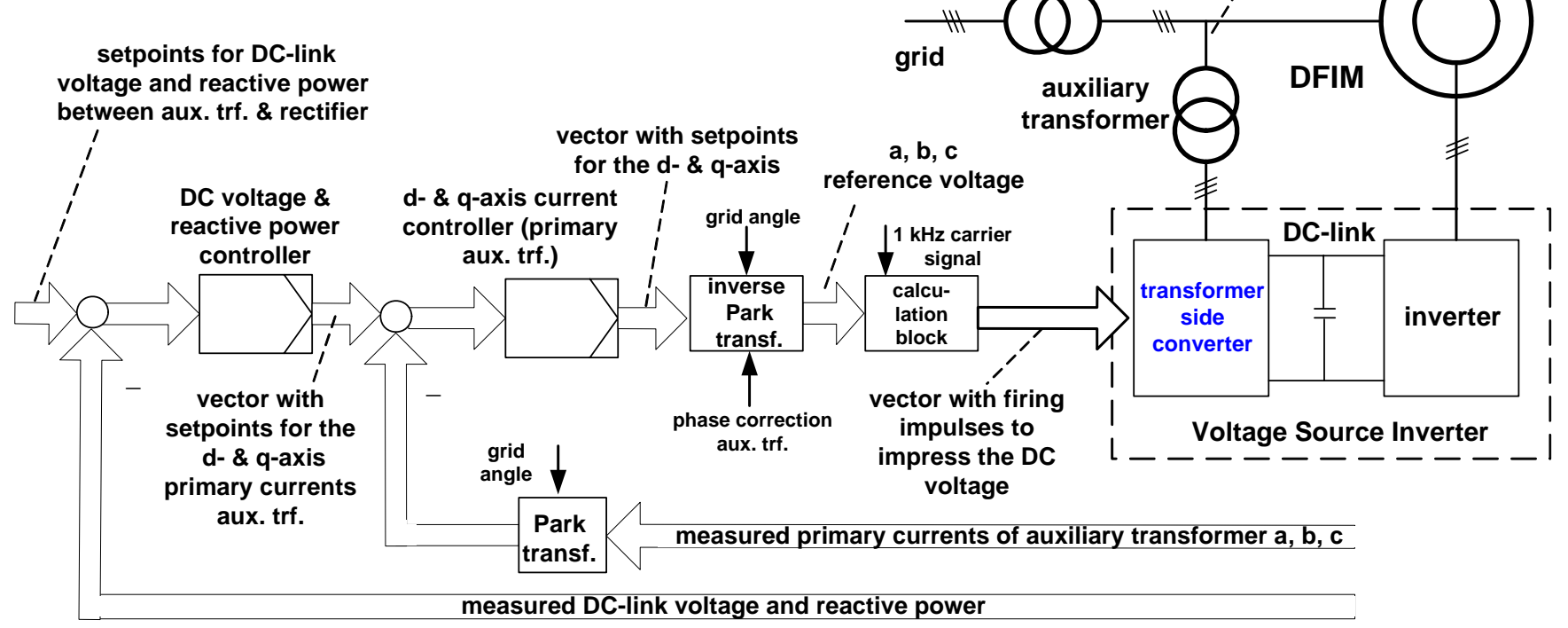

Fig. 8: Schematic control structure for the transformer-side converter 


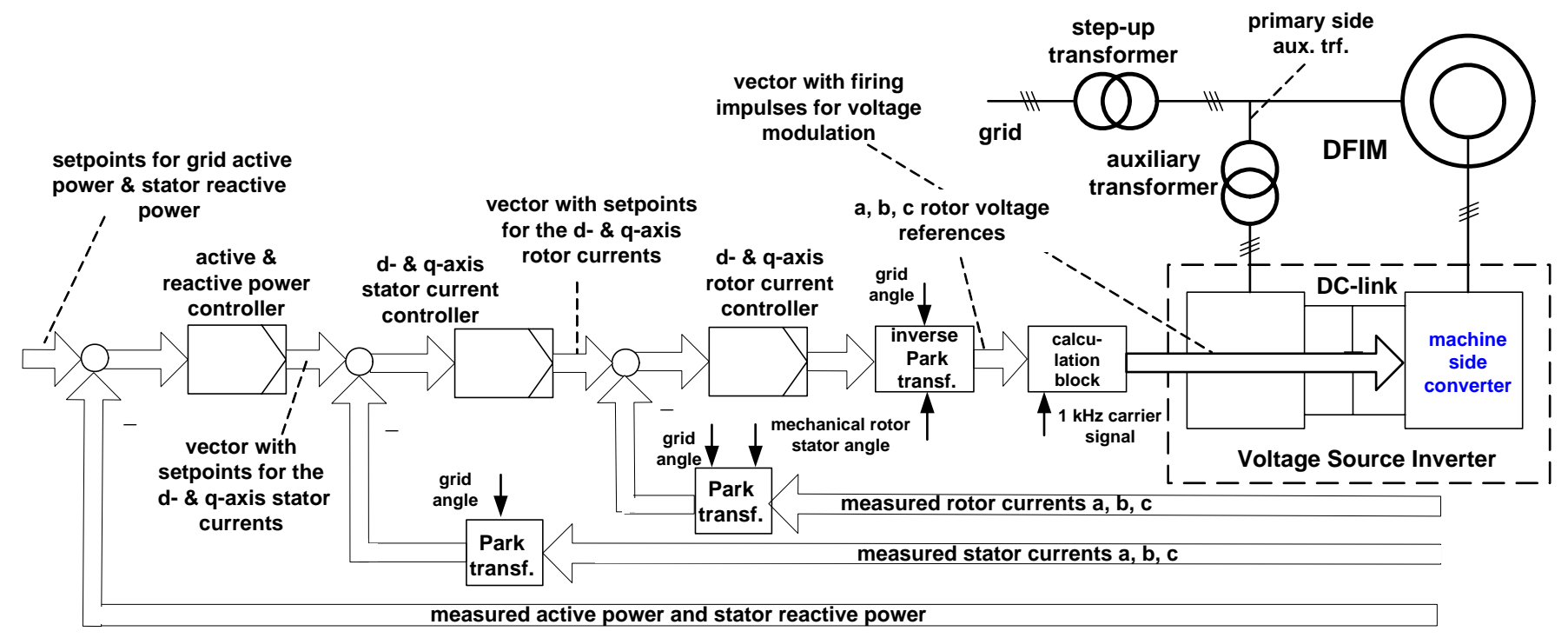

Fig. 9: Schematic control structure for the machine-side converter

On the machine-side of the VSI the over-all control structure in fig. 9 is a triple cascade control loop. The outer loop consists of two PI-controllers for the active grid power and the stator reactive power. The outputs of this master controller are setpoints for the d- and q-axis of the stator currents a, b, c for the middle control loop. The middle control loop is responsible for the control of two fictitious stator currents in d- and q-axis after using the Park transformation of the real measured stator currents a,b,c. The two outputs of this controller are the setpoints for rotor currents in d- and q-axis. Finally the inner controller is responsible for the rotor voltage references which are used to calculate the vector with impulses for the machine-side voltage modulation together with the carrier signal. The control structure for the machine-side converter in fig. 9 is valid for load operation after synchronization only. During start-up, synchronization or shut down a different and simpler control structure should be applied (single loop control structure for the rotor speed).

For the coupled simulations the turbine mode has been chosen with following nominal values for the model: SN = 142 MVA, $\mathrm{UN}=10.5 \mathrm{kV}, \mathrm{NN}=250 \mathrm{rpm}(12$ pole pairs $)$, JGeno $=1.4 \mathrm{e} 6 \mathrm{kgm} 2$, hnet $=150 \mathrm{~m}$, rotor speed $=90 \%$ of rated synchronous speed, max. rotor speed variation range $+/-20 \%$. As it can be seen in fig. 7 , the complete coupled system consisting of the hydraulic system, mechanical coupling \& mass inertia, the electrical machine with power converter and grid influence as well as the turbine governor and VSI control strategies shown in fig. 8 and 9 have been considered for the SIMSEN modeling. For the following simulations, different situations have been considered: normal load operation, disturbance rejection behaviour and the start-up of the DFIM with short-circuited stator.

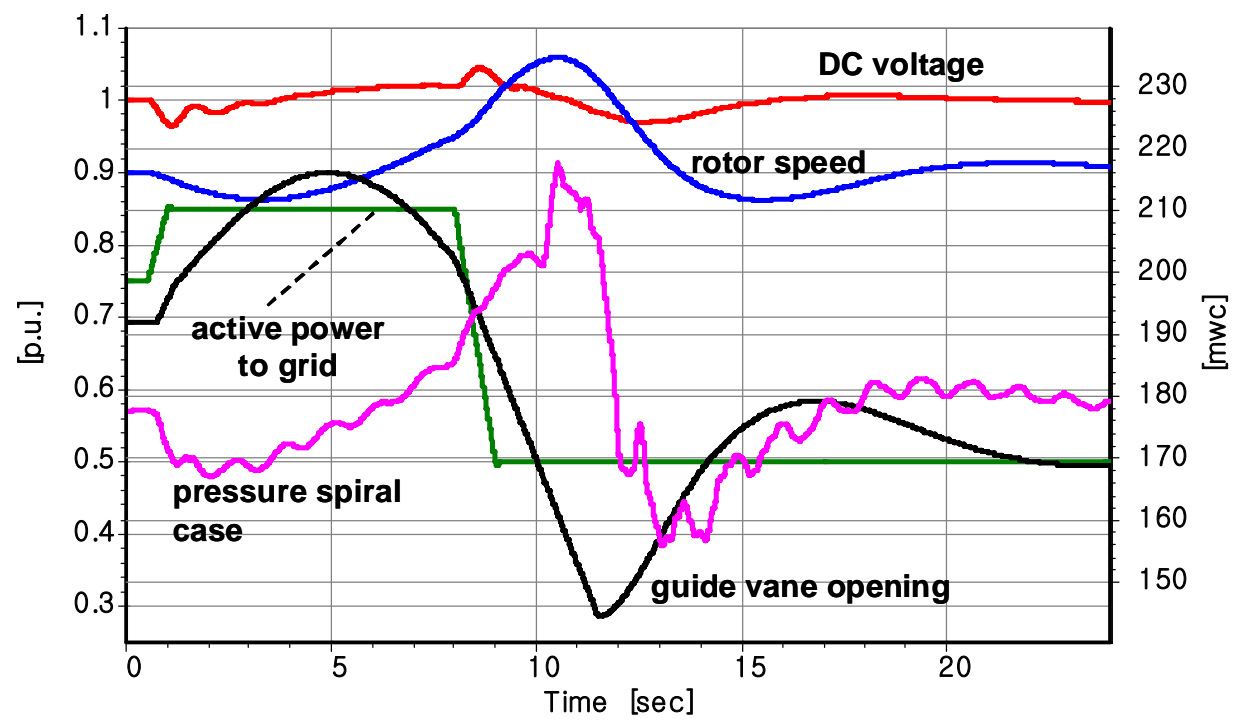

Fig. 10: Main variables during active power ramping (TM)

In fig. 10 active load ramping is shown including the influence on the rotor speed, the voltage in the DC-link of the VSI and also on the pressure in the spiral case and the guide vane opening. There is no significant bias between the load setpoint and the active power. The fast load ramping in turbine mode (TM) s possible due to the additional degree of freedom of variable speed units to temporarily change the rotor speed ("flywheel effect”). Therefore power loading and unloading dynamics can de-coupled from the slow distributor movement. The turbine controller operated in speed control mode slowly compensates the disturbance given by the fast load change impressed on the electrical side. However this applies only as long as the VSI is not limited in his 
designed operation range, i.e. fast load ramping by changing temporarily the rotor speed is only possible, if the transient speed deviations do not violate the admissible frequency range of the power converter. Due to the fact that especially in part load operation the stationary speed required to ensure optimum turbine efficiency is considerably lower than the synchronous speed, there is limited flexibility to benefit from the possibility for fast load changes in this case [12].

The next fig. 11 shows reactive power ramping. The reactive power variation has some small influence on the DC-link voltage, whereas the other main variables stay almost constant.

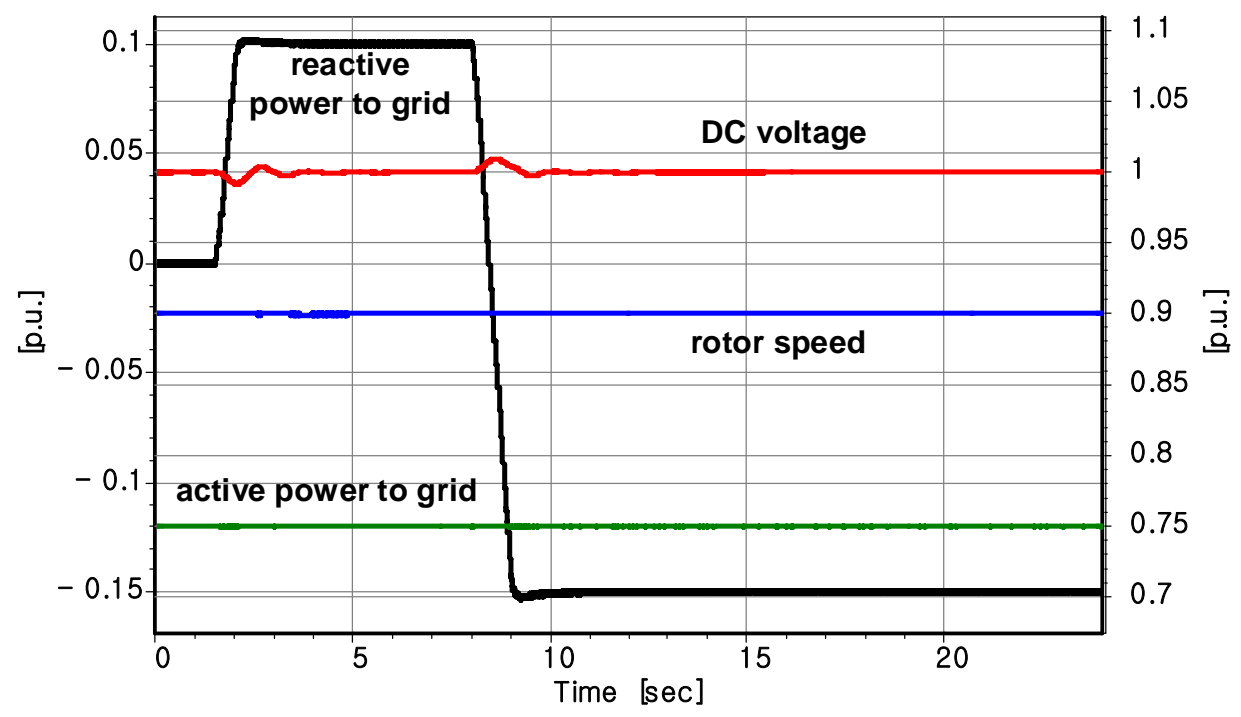

Fig. 11: Main variables during reactive power ramping (TM)

Fig. 12 shows the influence of a cavitating vortex rope in part load operation after a reduction of the active load. The cavitating vortex rope has been introduced in the hydraulic systems by inserting a pressure source (as indicated in fig.7). It should be pointed out that the fluctuations of speed, DC voltage and reactive power are a result of the active load ramping and not influenced by the pressure pulsations of approx. $1.3 \mathrm{~Hz}$ (31\% of the nominal speed). This simulation shows that the electrical system is decoupled from the hydraulic disturbance caused by the cavitating vortex rope.

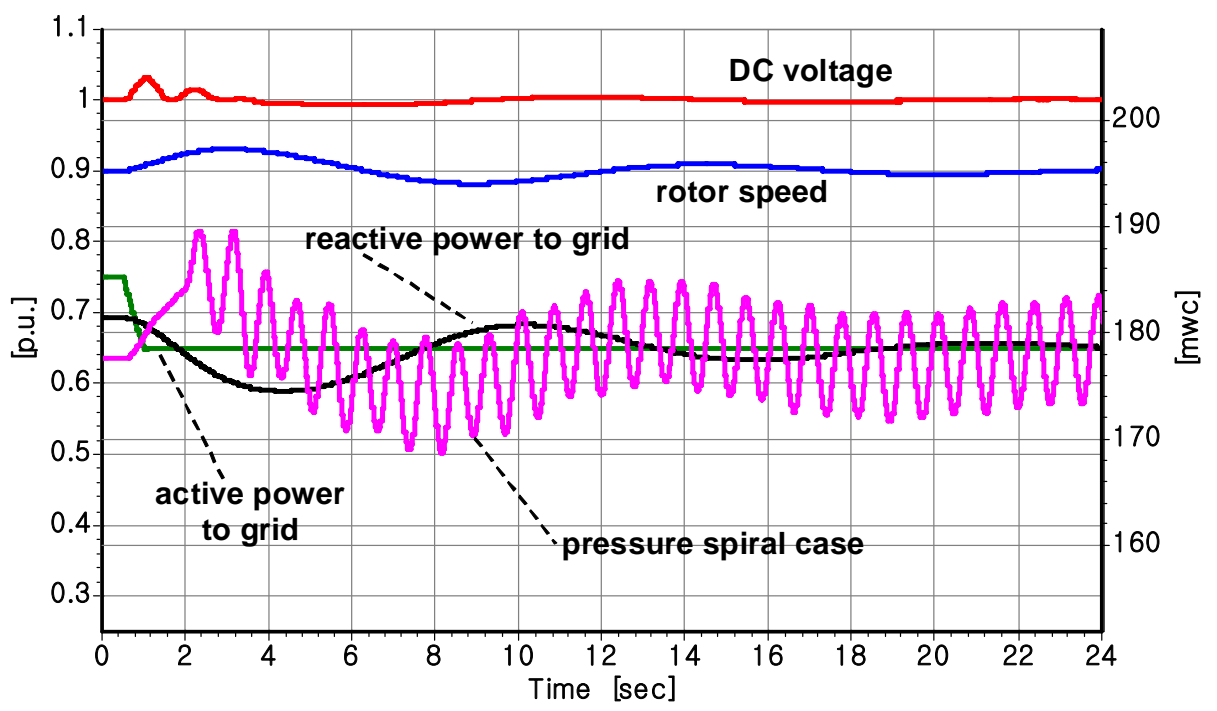

Fig. 12: Main variables during a hydraulic disturbance (cavitating vortex rope in TM)

The system behaviour after a three-phase short circuit on the primary side of the step-up transformer with a duration of 200ms is shown in fig. 13. In this case the short-circuit influences the hydraulic system and also the DC voltage, but with different settling times for the fluctuations. 


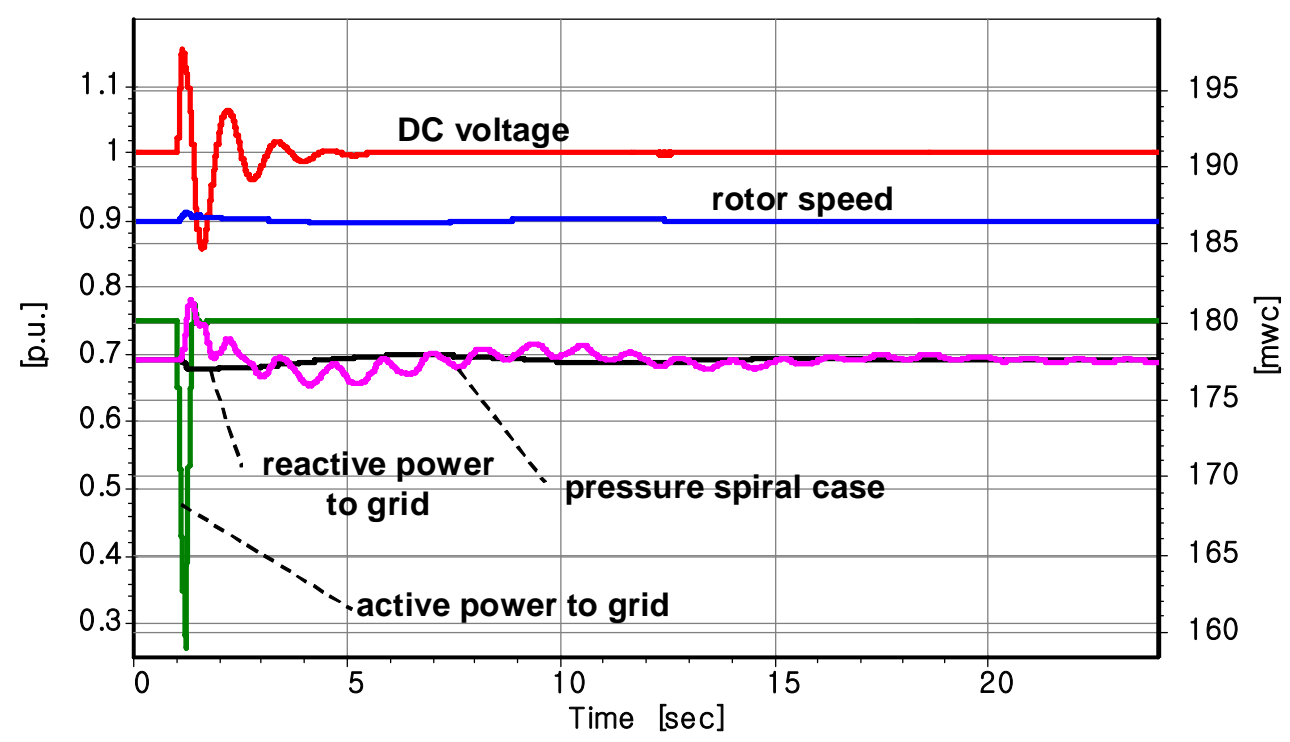

Fig. 13: Main variables after an electric disturbance (short circuit in TM)

The dynamic behaviour of the main variable in pumping mode with adjustable power is shown in fig. 14. In pump operation mode (PM) with active power control the rotor speed can not be controlled specifically and the turbine governor is in guide vanes position control mode. For practical applications the setpoint for the position of the guide vanes is not a constant value, but rather a head- and load-dependent value in order to obtain maximum efficiency. The pressure oscillations in the spiral case with a frequency of approx. $0,4 \mathrm{~Hz}$ reflects the dynamic pressure response of the upstream waterways with a length of $2 \mathrm{~km}$ excited by the discharge change due to rotor speed variation.

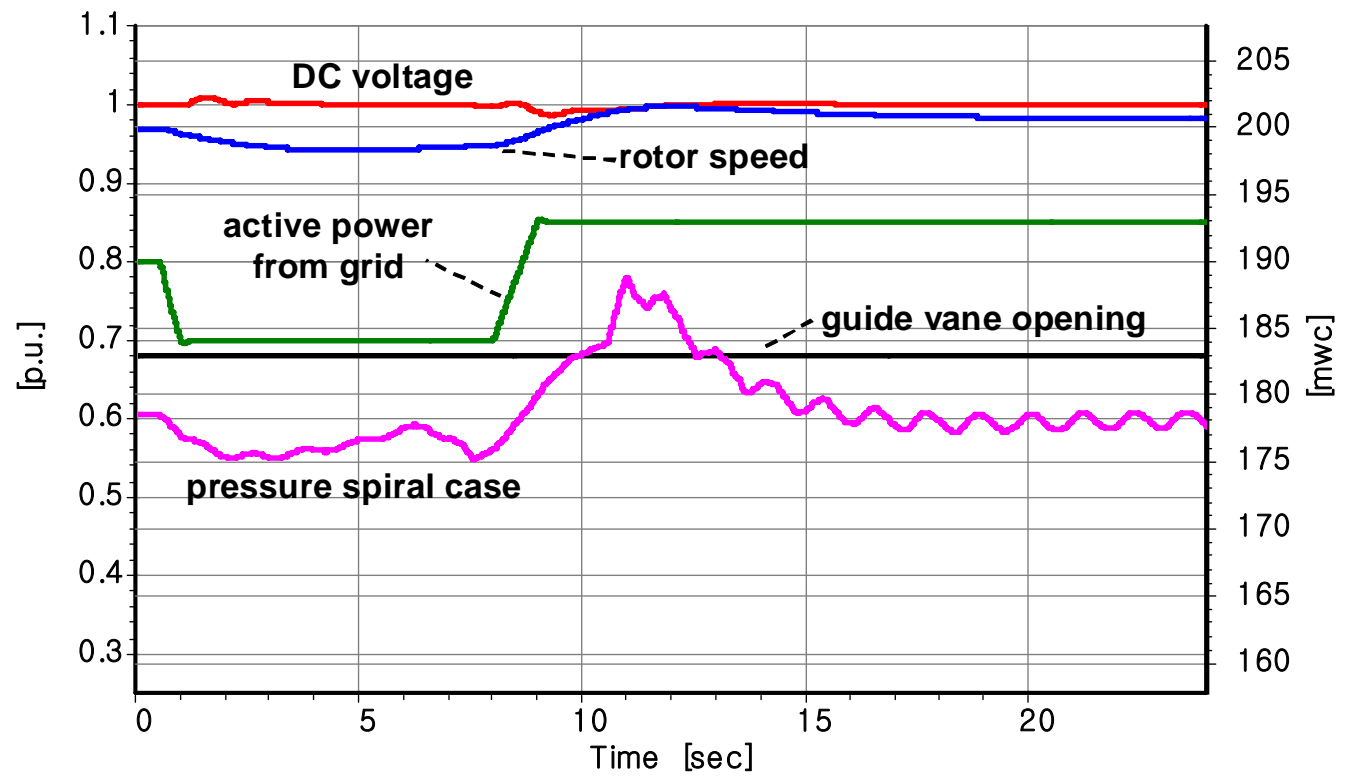

Fig. 14: Main variables in pump mode with adjustable power (PM)

As stated before a DFIM with a VSI does not need an SFC for start-up. The simulation in fig. 15 shows the start up of the DFIM with a short circuited stator. In this case the control structure of the machine-side converter in fig. 9 has to be changed to a single loop controller for the rotor speed. In both diagrams of fig. 15 the scaling of rotor speed and shaft torque are shown on the right hand y-axis. The left diagram shows the first 10 seconds of the start-up with high rotor and stator currents in the first 4 seconds (up to 3.5 times the rated value). The right diagram illustrates the complete start-up, which needs about 150 seconds. Such simulations are useful for the design of the electrical machine but also for detailed specification of the power converters. The simulation results shown in fig. 11 to 15 do not change, if a 3-level instead of a 2-level VSI is used. 

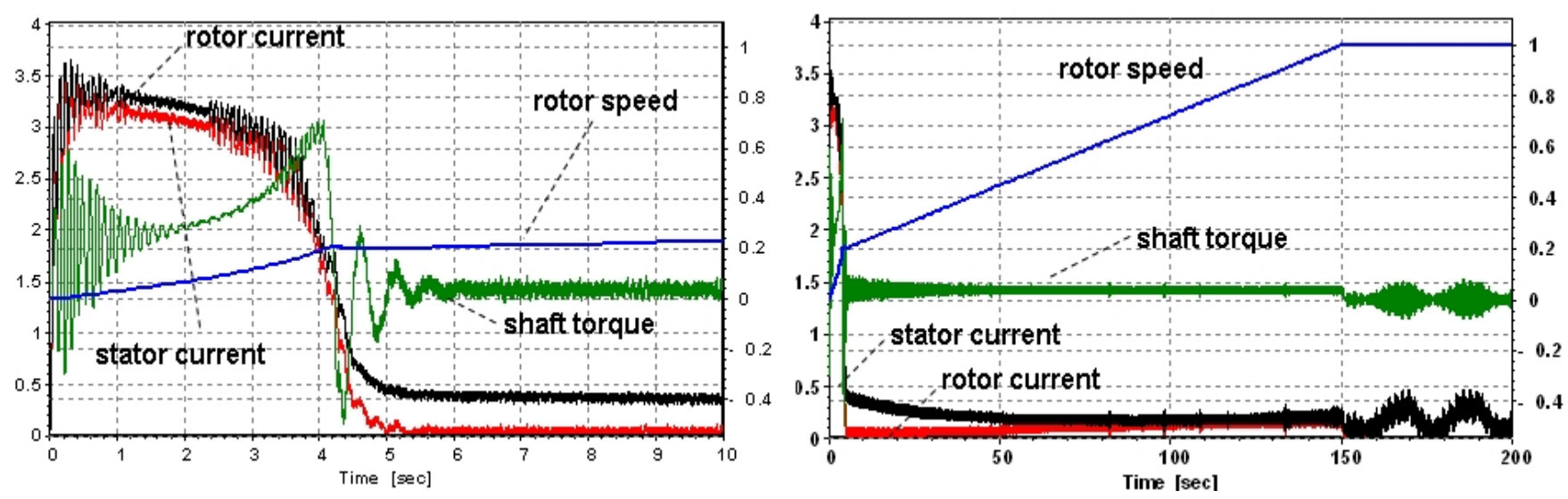

Fig. 15: Start-up of a DFIM with 2-level VSI with short-circuited stator

\section{Conclusions}

This paper comprises an overview on different configurations of variable speed units, pointing out their advantages \& disadvantages. Many important aspects for the basic design of a pumped storage power plant mentioned in the introduction can be investigated by dynamic off-line simulations including "what if" scenarios and considering the complete "water-to-wire" chain. Especially the dependencies and the limitations of all systems involved can be analyzed by coupled simulations of the hydraulic, mechanical, electrical systems including the related controls.

Fig. 16 shows an overview of dynamic effects versus the frequency range. If harmonic noise and electrical losses should be calculated and simulated only, then a complete coupled simulation is not necessary. It is sufficient to consider the electrical systems and the connected power electronics. Due to the modern semiconductors which allow pulse width modulation for firing and blocking in the range up to $1,5 \mathrm{kHz}$ a very small time step for simulation in the range of 1 microsecond is required. In this case the relevant frequency range is several $\mathrm{kHz}$ (right side of fig. 16).

The modeling and simulation of the power electronics can be skipped, if for instance the short circuit calculation for the electrical system is in the main focus for the analysis. In this case the interesting frequency range is between 50 and $200 \mathrm{~Hz}$. The simulation time step is then in the range of milliseconds. The last scenario shown on the left side of fig. 16 is relevant if effects like interactions of the water ways with turbine controller or the DC or AC excitation systems should be considered. In this case a coupled simulation of all systems is necessary. Here the typical range of interesting frequencies is between 0,2 and $10 \mathrm{~Hz}$. Another example would be the rotor oscillation in the magnetic field.
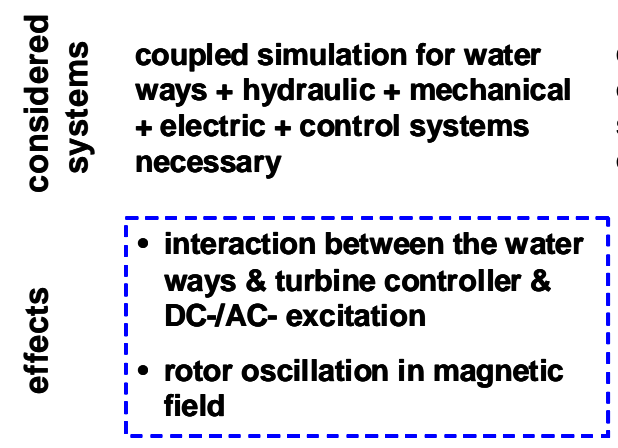
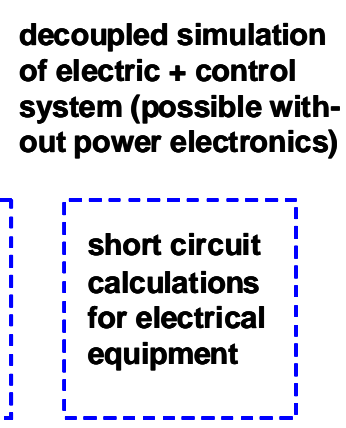

\section{decoupled simulation of electric systems + controls + power electronics}

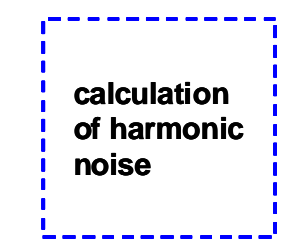

\begin{tabular}{|c|c|c|c|c|}
\hline $0,1 \mathrm{~Hz}$ & 1 & 10 & 100 & $1000 \quad 10000 \mathrm{~Hz}$ \\
\hline
\end{tabular}

Fig. 16: Dynamic analysis versus frequency range

Based on its longtime knowledge on pumped storage plant and control system technologies, Voith Hydro can offer a custommized solution minimizing cost and exploiting the maximum usage of pump-turbine units for pump storage projects. A well suited solution considering given project constraints can be developed by means of comparative investigations of variants at a very early stage of the project development. This also helps to reduce the risks in the overall basic design.

Such dynamic simulations require next to the knowledge of the subsystems involved also a comfortable and reliable tool providing adequate capabilities for the simulation of interactions between electrical part (including power converters), control blocks and hydraulic systems. The SIMSEN advanced simulation technology is a very good approach to fulfill these ambitious demands. 


\section{References}

[1] Hauff, C., Klein, J., Jaeger, E.-U. and Scherer, T., "Modern Pump Turbines Features of Adjustable Speed Machines,” 20th IAHR Symposium on Hydraulic Machinery and Systems, 2000, Charlotte, USA.

[2] Nicolet, C., Sapin, A., Simond, J.-J., Prenat, J.-E. and Avellan, F., "A new tool for the simulation of dynamic behaviour of hydroelectric power plants,” Proceedings of the 10th International Meeting of WG1, IAHR, Trondheim, Norway, June 26-28, 2001.

[3] Simond, J.-J., Allenbach, P., Nicolet, C. and Avellan, F., "Simulation tool linking hydroelectric production sites and electrical networks,” Proceedings of the 27th Int. Conf. on Electrical Machines, ICEM, Chania, Greece, September 2-5, 2006.

[4] Koutnik, J., Kahler, J. and Krüger, K., "Pump-storage with adjustable speed - Simulative comparison of different variants," HydroVision conference, July 14-18, 2008, Sacramento, USA.

[5] Förster, H and Wagnitz, R., "Ein Frequenzumrichter für die Speicherpumpe im Pumpspeicherkraftwerk Forbach der Badenwerke, ” AEG Project Report, online, http://aeg-ibo.com/deutsch/energieerzeugung_generatoranlagen_notstrom_usv.htm, 1993.

[6] Simond, J.-J., Sapin, A., Tu Xuan, M., Wetter, R. and Burmeister, P., "12-pulse LCI synchronous drive for a 20MW compressor - Modelling, Simulation and Measurements," Industry Applications Conference 2005, Volume 4, pp. 2302-2308.

[7] Wu, B., "High-Power Converters and AC Drives,” IEEE Press, Wiley - Interscience, New Jersey, 2006.

[8] Bernet, S., "State of the art and recent developments of MV converters," Seminar of the PELINCEC working group 2 - New high voltage semiconductor devices and its application for MV converters, online slides,

http://pelincec.isep.pw.edu.pl/wg2_seminar.htm, Warsaw, Poland, 2003.

[9] Caroll, E. and Galster, N., "IGBT or IGCT: Considerations for very high power applications," Forum Européen des Semiconducteurs de Puissance, 1997.

[10] Hodder, A., "Double-fed asynchronous motor-generator equipped with a 3-level VSI cascade," PhD Thesis No. 2939, École Polytechnique Fédérale de Lausanne, 2004.

[11] TM GE Automation Systems, "MV Drive Evolution - Comparisons and Feature-Benefits," Toshiba-Mitsubishi GE Automation Systems, USA, Brochure, online, www.tmge.com/main/library.php, 2005.

[12] Kopf, E., Brausewetter, S., Giese, M. and Moser, F., “Optimized Control Strategies for Variable Speed Machines," 22nd IAHR Symposium on Hydraulic Machinery and Systems, 2004, Stockholm, Sweden. 